\title{
Prognosis and outcome of patients with acute myeloid leukemia based on FLT3-ITD mutation with or without additional abnormal cytogenetics
}

\author{
SHANDONG TAO ${ }^{1,2^{*}}$, CHUNLING WANG ${ }^{1,2^{*}}$, YUE CHEN $^{1,2}$, YUAN DENG $^{1,2}$, LIXIAO SONG $^{1,2}$, \\ YUYUE SHI ${ }^{1,2}$, LANLAN LING ${ }^{1,2}$, BANGHE DING ${ }^{1,2}$, ZHENGMEI HE ${ }^{1,2}$ and LIANG YU ${ }^{1,2}$ \\ ${ }^{1}$ Department of Hematology, The Affiliated Huaian No. 1 People's Hospital of Nanjing Medical University, Huai'an, \\ Jiangsu 223300; ${ }^{2}$ Key Laboratory of Hematology of Nanjing Medical University, Nanjing, Jiangsu 210029, P.R. China
}

Received May 7, 2019; Accepted October 10, 2019

DOI: 10.3892/ol.2019.11051

\begin{abstract}
The FMS-like tyrosine kinase 3-internal tandem duplication (FLT3-ITD) gene mutation is present in $\sim 20 \%$ of patients with de novo acute myeloid leukemia (AML). Patients with an FLT3-ITD mutation have a poor prognosis. However, the prognostic function of FLT3-ITD combined with other cytogenetic abnormalities are not clear. In the present study, a retrospective analysis of 103 newly diagnosed patients with AML was performed. The results revealed that the overall survival (OS) and recurrence-free survival (RFS) times were significantly longer in patients with an FLT3-ITD mutation combined with other favorable risk genes, compared with in those patients with a single FLT3-ITD mutation $(\mathrm{P}=0.0361$ and $\mathrm{P}=0.0426$ ). Sorafenib combined with chemotherapy significantly improved the overall response rate (ORR) when compared with mono-chemotherapy $(\mathrm{P}=0.039)$, but no significant differences were observed in the OS and RFS. In conclusion, favorable-risk cytogenetics may improve the clinical outcomes of patients with FLT3-ITD-mutated AML, but adverse-risk cytogenetics may not further worsen
\end{abstract}

Correspondence to: Professor Liang Yu, Department of Hematology, The Affiliated Huaian No. 1 People's Hospital of Nanjing Medical University, 1 Huang River Road West, Huai'an, Jiangsu 223300, P.R. China

E-mail: yuliangha@163.com

*Contributed equally

Abbreviations: AML, acute myeloid leukemia; CR, complete remission; OS, overall survival; RFS, recurrence free survival; NPM1, nucleophosmin 1; FLT3-ITD, FMS-like tyrosine kinase 3-internal tandem duplication; FLT3-TKD, FMS-like tyrosine kinase 3-tyrosine kinase domain

Key words: acute myeloid leukemia, FMS-like tyrosine kinase 3-internal tandem duplication mutation, prognosis, favorable-risk cytogenetics, adverse-risk cytogenetics the prognosis. Sorafenib combined with chemotherapy may increase the ORR but would not result in a longer OS and RFS.

\section{Introduction}

Acute myeloid leukemia (AML), a highly heterogeneous disease, is characterized by uncontrolled proliferative blasts (1). At present, the treatment of AML is challenging due to patient characteristics and intrinsic biological factors (2). FMS-like tyrosine kinase 3 (FLT3) gene mutations are present in $\sim 30 \%$ of patients with de novo AML; internal tandem duplication (ITD) mutations in the juxtamembrane domain of FLT3 account for two-thirds of FLT3 mutations (3). Patients with an FLT3-ITD mutation have a poor prognosis, with a shorter remission duration and higher relapse rates compared with patients with FLT3-unmutated AML (3). The FLT3-ITD mutation usually coexists with other gene mutations or fusion genes; it has been reported that the prognosis of patients with FLT3-ITD and nucleophosmin (NPM1) double mutations was better compared with that in those with a FLT-ITD mono-mutation (4). Previously, research has indicated that the occurrence of FLT3-tyrosine kinase domain (TKD) and NPM1 double mutations were a highly favorable prognostic factor (5). However, the prognostic function of FLT3-ITD combined with other gene mutations or fusion genes is not clear.

Small molecule inhibitors that target FLT3, including sorafenib and midostaurin, have been revealed to be effective in the treatment of patients with FLT3-ITD-mutated AML; the multitargeted kinase inhibitor midostaurin combined with standard chemotherapy prolonged the overall survival (OS) and event-free survival times in patients with FLT3-mutated AML who were in remission after consolidation therapy and entered a maintenance phase compared with patients who received placebo plus chemotherapy (2). In addition, a novel irreversible FLT3 inhibitor, FF-10101, also demonstrated excellent efficacy against FLT3-mutated AML cells (6). However, the long-term efficacy of these novel FLT3 inhibitors, and whether they may be used as substitutes for hematopoietic stem cell transplantation (HSCT) for patients with FLT3-ITD mutations is currently unclear. 
In the present study, a retrospective analysis was performed to examine the complete remission (CR), relapse and survival of newly diagnosed patients with FLT3-ITD-mutated AML with or without other gene mutations or fusion genes, and the effect of sorafenib was evaluated in patients with FLT3-ITD mutated AML.

\section{Patients and methods}

Patient population. Adult patients with AML ( $\mathrm{n}=103$; age range 18-87 years; mean age, 50 years; 62 men and 41 women) diagnosed between January 2013 and June 2018 at Huai'an No. 1 People's Hospital, Nanjing Medical University (Nanjing, China), including 23 patients harboring an FLT3-ITD mutation, who were treated with different treatment regimens, were retrospectively included in the present study. A total of 45 patients were revealed to be alive at the time of data collection. Patients with acute promyelocytic leukemia were excluded. The study was ethically approved by the Institutional Review Committee of Huai'an No. 1 People's Hospital, and written informed consent was obtained from all patients. The diagnosis of AML was established according to the criteria of the WHO classification (7), including clinical presentations and morphological, immunophenotype and recurrent cytogenetic abnormalities. All patients were analyzed based on their response to induction therapy, CR, relapse, OS and recurrence-free survival (RFS) rates.

Detection of FLT3-ITD and other associated genes. Multiple markers for the diagnosis of AML were identified, along with gene mutations, including AML1-ETO, CBFB/MYH11, MLL/AF6, MLL/AF9, MLL/ENL, dupMLL, DEK/CAN, EVI1, HOX11, BCR/ABL, FLT3-ITD, NPM1, CEBPA, C-kit and $D N M T 3 A$. Fusion genes were detected using multiple nested RT-PCR. Total RNA was extracted using Omega whole-blood RNA extraction kit (cat. no. R6616-02; Omega Bio-Tek, Inc., Norcross, GA, USA). Nested RT-PCR detection method and reaction system were used as previously reported $(8,9)$. AML-associated mutated genes were detected using high-throughput sequencing technology. Total DNA was extracted using whole blood DNA extraction kit (cat. no. D3392-02; Omega Bio-Tek, Inc.), and genetic mutations were detected by Kindstar Global Medical Laboratory Center (http://www.kindstar.com.cn/kindstar/cn/platform. html) using Sanger sequencing, as previously described $(9,10)$. Bone marrow samples were used for this, and each patient signed a bone marrow puncture written informed consent form.

Treatment regimens. All 103 patients received a ' $3+7$ ' chemotherapy regimen $\left(60 \mathrm{mg} / \mathrm{m}^{2} /\right.$ day daunorubicin or $10 \mathrm{mg} / \mathrm{m}^{2} /$ day idarubicin for 3 days, and $100 \mathrm{mg} / \mathrm{m}^{2} /$ day cytarabine for 7 days with a 24-h intravenous pump maintenance). Out of the 23 patients harboring FLT3-ITD mutations, 7 were treated with sorafenib combined with a ' $3+7$ ' chemotherapy regimen for induction therapy. If the interim bone marrow (BM) examination, which was performed between days 14 and 21 of induction therapy, revealed residual leukemic blasts, a second course of induction chemotherapy comprising cytarabine (100 $\mathrm{mg} / \mathrm{m}^{2} /$ day) plus $2 \mathrm{mg} / \mathrm{m}^{2} /$ day homoharringtonine was administered for 5 days. Patients who achieved CR usually received four to six courses of consolidation chemotherapy or allo-HSCT. Consolidation chemotherapy regimens included high-dose cytarabine ( $3 \mathrm{~g} / \mathrm{m}^{2}$ twice a day on days 1 to 3 ) or intermediate-dose cytarabine $\left(1 \mathrm{~g} / \mathrm{m}^{2}\right.$ for 4 days $)$ plus daunorubicin ( $45 \mathrm{mg} / \mathrm{m}^{2} /$ day for 3 days) or mitoxantrone $\left(4 \mathrm{mg} / \mathrm{m}^{2} /\right.$ day for 3 days). The patients who achieved CR whose induction regimen contained sorafenib with chemotherapy received a continuous maintenance of sorafenib during the chemotherapy interval. Allo-HSCT was performed in patients who achieved CR at the discretion of the attending physician, usually following two courses of consolidation chemotherapy; however, decisions were often made on the basis of the patients' willingness, disease status, risk classification, human leukocyte antigen (HLA) matching and financial status.

Evaluation. CR was defined according to the standard criteria of $<5 \%$ blasts in BM. Hematologic recovery was measured in terms of the absolute neutrophil $\left(>1 \times 10^{9} / 1\right)$ and platelet $\left(>100 \times 10^{9} / 1\right)$ counts in the peripheral blood. Clinical recurrence following $\mathrm{CR}$ was defined as the presence of $\geq 5 \%$ blasts in BM or re-appearance of leukemic blasts in the peripheral blood, or the presence of extramedullary disease. OS and RFS were calculated from the date of diagnosis. An event was defined as induction therapy failure, relapse following CR or mortality from any cause. Relapse was evaluated in patients who achieved CR using a cumulative incidence function with respect to competing risks.

Statistical analysis. Statistical analysis was performed using SPSS 24.0 (IBM Corp., Armonk, NY, USA) and data were presented as the means \pm standard deviation/standard error of the mean. Pearson $\chi^{2}$ survival distributions were estimated using the Kaplan-Meier method and the differences were compared using the log-rank (Mantel-Cox) test. Categorical variables were compared using Pearson $\chi^{2}$ or Fisher Exact test. $\mathrm{P}<0.05$ was considered to indicate a statistically significant difference.

\section{Results}

Patient characteristics. A total of 103 patients with AML were investigated during this period, 23 (22.3\%) of which had an FLT3-ITD mutation. The median age of the entire cohort was 50 years old (age range, 18-87 years); 31 (30.1\%) patients were aged $\geq 60$ years. Patient characteristics and FLT3-ITD mutation statuses are summarized in Table I. Eleven out of $23(47.83 \%)$ patients had both NPMI and FLT3-ITD mutation. High leukocyte counts usually occurred in patients with FLT3-ITD mutations. In addition to NPM1, other gene mutations combined with the FLT3-ITD mutation were observed as follows: CEBPA (2/23, 8.69\%), AML1-ETO (1/23, 4.35\%), EVII (1/23, 4.35\%), HOX11 (5/23, 21.74\%) and dupMLL $(2 / 23$, $8.96 \%$ ) mutations.

Treatment response. All 103 patients received a standard ' $3+7$ ' regimen of induction chemotherapy. There were no significant differences in the $\mathrm{CR}$ rate between the FLT3-ITD-positive and FLT3-ITD-negative groups (65.22 vs. $62.5 \%$; $\mathrm{P}=0.812$; Table II). The overall response rate (ORR) was $78.26 \%$ for the FLT3-ITD-positive group and $86.25 \%$ 
Table I. Patient characteristics.

\begin{tabular}{|c|c|c|c|c|}
\hline Characteristics & Total $(n=103)$ & $F L T 3-I T D(+)(\mathrm{n}=23)$ & FLT3-ITD $(-)(\mathrm{n}=80)$ & P-value \\
\hline Age (years), median (range) & $50(18-87)$ & $51(18-87)$ & $49.5(18-76)$ & 0.138 \\
\hline$<60$ years, no. $(\%)$ & $82(79.61)$ & $15(65.22)$ & $57(71.25)$ & \\
\hline$\geq 60$ years, no. $(\%)$ & $31(20.39)$ & $8(34.78)$ & $23(28.75)$ & 0.578 \\
\hline Male, no. (\%) & $62(60.19)$ & $12(52.17)$ & $50(62.50)$ & \\
\hline Female, no. (\%) & $41(39.81)$ & $11(47.83)$ & $30(37.50)$ & 0.372 \\
\hline WBC $\left(x 10^{9} / 1\right)$, median (range) & $15.29(0.63-257.0)$ & $40.44(1.55-257.0)$ & $9.71(0.63-211.0)$ & $<0.001^{\mathrm{a}}$ \\
\hline HB (g/l), median (range) & $74(40-134)$ & $65(46-127)$ & $79.5(40-134)$ & 0.842 \\
\hline PLT $\left(x 10^{9} / 1\right)$, median (range) & $44.0(1-461)$ & $48(4-219)$ & $41.5(1-461)$ & 0.290 \\
\hline \multicolumn{5}{|l|}{ Cytogenetics, no. (\%) } \\
\hline Abnormal karyotype & $44(42.72)$ & $5(21.74)$ & $39(48.75)$ & \\
\hline Normal karyotype & $59(57.28)$ & $18(78.26)$ & $41(51.25)$ & $0.021^{\mathrm{a}}$ \\
\hline \multicolumn{5}{|l|}{ Other abnormal genes no. $(\%)$} \\
\hline$N P M 1$ & $18(17.48)$ & $11(47.83)$ & $7(13.75)$ & $0.0001^{\mathrm{a}}$ \\
\hline CEBPA & $18(17.48)$ & $2(8.69)$ & $16(20.00)$ & 0.208 \\
\hline AML1/ETO & $17(16.50)$ & $1(4.35)$ & $16(20.00)$ & 0.074 \\
\hline EVII & $9(8.74)$ & $1(4.35)$ & $8(10.00)$ & 0.397 \\
\hline HOX11 & $9(8.74)$ & $5(21.74)$ & $4(5.00)$ & $0.012^{\mathrm{a}}$ \\
\hline$C$-kit/D816V & $7(6.79)$ & $0(0)$ & $7(8.75)$ & 0.141 \\
\hline Dup MLL & $3(2.91)$ & $2(8.96)$ & $1(1.25)$ & 0.061 \\
\hline CBFB/MYHII & $2(1.94)$ & $0(0)$ & $2(2.50)$ & 0.281 \\
\hline$B C R / A B L$ & $2(1.94)$ & $0(0)$ & $2(2.50)$ & 0.443 \\
\hline$D E K / C A N$ & $2(1.94)$ & $0(0)$ & $2(2.50)$ & 0.443 \\
\hline$M L L / A F 6$ & $2(1.94)$ & $0(0)$ & $1(1.25)$ & 0.590 \\
\hline$M L L / A F 9$ & $2(1.94)$ & $0(0)$ & $2(2.50)$ & 0.443 \\
\hline$M L L / A F 10$ & $1(0.97)$ & $0(0)$ & $1(1.25)$ & 0.590 \\
\hline$M L L / E L L$ & $2(1.94)$ & $0(0)$ & $2(2.50)$ & 0.443 \\
\hline NuP98/HOxC11 & $1(0.97)$ & $0(0)$ & $1(1.25)$ & 0.338 \\
\hline
\end{tabular}

PLT, platelet; WBC, white blood cell count; HB, hemoglobin; FLT3-ITD, FMS-like tyrosine kinase 3-internal tandem duplication mutation;

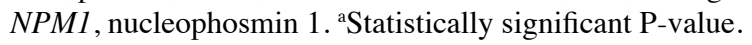

for the FLT3-ITD-negative group $(\mathrm{P}=0.351)$. The relapse rate was significantly higher in the FLT3-ITD-positive group compared with the FLT3-ITD-negative group (34.78 vs. $15.0 \%$; $\mathrm{P}=0.034)$. One patient harboring an FLT3-ITD mutation received allo-HSCT, and still had CR status 6 months later. In the FLT3-ITD-negative group, 10 patients received autologous stem cell transplantation, 3 received allo-HSCT and 4 received micro-transplantation (11). Out of the 23 patients harboring FLT3-ITD mutations, 7 were treated with sorafenib combined with chemotherapy for induction therapy and 16 with mono-chemotherapy. The ORR was significantly higher in the sorafenib plus chemotherapy group compared with in the mono-chemotherapy group ( $\mathrm{P}=0.039$; Table III), but no significant differences in the $\mathrm{CR}$ and relapse rates were observed between these two groups.

Sorafenib plus chemotherapy did not improve the OS and RFS of patients with FLT3-ITD-mutations who did not receive allo-HSCT. Patients with FLT3-ITD-mutated AML were associated with a poor prognosis due to a high relapse rate (12). In the present study, 103 patients with AML who completed at least one cycle of induction therapy were analyzed, and the OS and RFS rates in 23 patients harboring an FLT3-ITD mutation were revealed to be significantly lower compared with those in patients with FLT3-ITD-unmutated AML $(\mathrm{P}=0.0295$ and $\mathrm{P}=0.0238$, respectively; Fig. $1 \mathrm{~A}$ and $\mathrm{B})$. A number of FLT3 inhibitors have been revealed to specifically inhibit the proliferation of leukemia cells in preclinical models. However, first-generation FLT3 inhibitors in patients with relapsed/refractory FLT3-mutated AML revealed transient reductions in the number of blasts in the blood and bone marrow, but rarely CR $(13,14)$. In the present study, 7 patients with an FLT3-ITD mutation received sorafenib plus chemotherapy, and their ORR was revealed to be higher compared with those of patients with FLT3-ITD-unmutated AML, but no significant differences in the OS and RFS were observed between the mono-chemotherapy and sorafenib plus chemotherapy groups $(\mathrm{P}=0.2735$ and $\mathrm{P}=0.7302$, respectively; Fig. 1C and D). These results indicate that sorafenib was not able to improve the long-term follow-up results of patients harboring an FLT3-ITD mutation, and should receive allo-HSCT as soon as possible. 
Table II. Treatment response between the FLT3-ITD positive group and FLT3-ITD negative group.

\begin{tabular}{|c|c|c|c|c|}
\hline Treatment & Total $(n=103)$ & $F L T 3-I T D(+)(\mathrm{n}=23)$ & $F L T 3-I T D(-)(\mathrm{n}=80)$ & P-value \\
\hline \multicolumn{5}{|l|}{ Therapy regimens } \\
\hline Mono-chemotherapy, no. (\%) & $96(93.2)$ & $16(69.57)$ & $80(100.0)$ & \\
\hline Sorafenib + chemotherapy, no. $(\%)$ & $7(6.8)$ & $7(30.43)$ & $0(0)$ & \\
\hline \multicolumn{5}{|l|}{ Treatment response } \\
\hline NR, no. $(\%)$ & $16(15.53)$ & $5(21.74)$ & $11(13.75)$ & 0.351 \\
\hline PR, no. (\%) & $22(21.34)$ & $3(13.04)$ & $19(23.75)$ & 0.269 \\
\hline CR, no. $(\%)$ & $65(63.12)$ & $15(65.22)$ & $50(62.5)$ & 0.812 \\
\hline ORR, no. (\%) & $87(84.46)$ & $18(78.26)$ & $69(86.25)$ & 0.351 \\
\hline Relapse, no. (\%) & $20(19.42)$ & $8(34.78)$ & $12(15.00)$ & $0.034^{\mathrm{a}}$ \\
\hline \multicolumn{5}{|l|}{ Post-remission treatment } \\
\hline Autologous transplantation (\%) & $10(9.71)$ & $0(0)$ & $10(12.5)$ & \\
\hline Allogeneic transplantation (\%) & $4(3.88)$ & $1(4.35)$ & $3(3.75)$ & \\
\hline Micro transplantation (\%) & $4(3.88)$ & $0(0)$ & $4(5.00)$ & \\
\hline
\end{tabular}

NR, no response; PR, partial remission; CR, complete remission; ORR, overall response rate; FLT3-ITD, FMS-like tyrosine kinase 3-internal

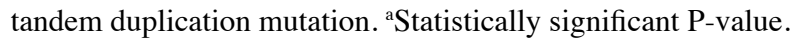

Table III. Treatment of patients with FMS-like tyrosine kinase 3-internal tandem duplication mutation mutations.

\begin{tabular}{|c|c|c|c|c|}
\hline Treatment response $(\%)$ & Total $(n=23)$ & Mono-chemotherapy $(\mathrm{n}=16)$ & Sorafenib plus chemotherapy $(n=7)$ & P-value \\
\hline NR, no. & $5(21.74)$ & $5(31.25)$ & $0(0)$ & $0.039^{\mathrm{a}}$ \\
\hline PR, no. & $3(13.04)$ & $2(12.50)$ & $1(14.28)$ & 0.906 \\
\hline CR, no. & $15(65.22)$ & $9(56.25)$ & $6(85.71)$ & 0.172 \\
\hline OR, no. & $18(78.26)$ & $11(68.75)$ & $7(100.00)$ & $0.039^{\mathrm{a}}$ \\
\hline Relapse, no. & $8(34.78)$ & $4(25.00)$ & $4(57.14)$ & 0.136 \\
\hline
\end{tabular}

NR, no response; PR, partial remission; CR, complete remission; OR, overall response. 'Statistically significant P-value.

Table IV. FLT3-ITD mutation together with other genetic abnormalities in 75 patients with acute myeloid leukemia.

\begin{tabular}{lcccr}
\hline FLT3-ITD status & $\begin{array}{c}\text { Total } \\
(\mathrm{n}=75)(\%)\end{array}$ & $\begin{array}{c}\text { Favourable-risk } \\
\text { genes }(\mathrm{n}=46)(\%)\end{array}$ & $\begin{array}{c}\text { Adverse-risk } \\
\text { genes }(\mathrm{n}=22)(\%)\end{array}$ & $\begin{array}{c}\text { Neither }(\mathrm{n}=7) \\
\text { P-value }\end{array}$ \\
\hline FLT3-ITD $(+)$ & $23(30.67)$ & $11(23.91)$ & $5(22.73)$ & $7(100.0 \%)$ \\
FLT3-ITD $(-\mathrm{a})$ & $52(69.33)$ & $35(76.09)$ & $17(77.27)$ & 0
\end{tabular}

Neither, only FLT3-ITD mutation not together with other genes; FLT3-ITD, FMS-like tyrosine kinase 3-internal tandem duplication mutation.

Outcome of patients harboring an FLT3-ITD mutation combined with other gene mutations or fusion genes. The prognosis and outcome in patients harboring an FLT3-ITD mutation combined with other mutations or fusion genes are unclear. In the present study, two groups of patients harboring an FLT3-ITD mutation combined with favorable-risk genes (FRGs; defined as the presence alone of the AML1-ETO, CBFB/MYH11 fusion gene or NPM1 mutation, and $C E B P A$ double mutation) or adverse-risk genes (DRGs; defined as multiple gene mutations, specific genes associated with a poor prognosis for AML, and complex chromosomal karyotype abnormalities, for example FLT3-ITD, C-kit and DNMT3A mutations) were analyzed. A total of 75 patients with genetic abnormalities received standard regimens of chemotherapy. The survival of all patients with an FLT3-ITD mutation combined with FRGs were compared with those of patients with an FLT3-ITD mutation combined with DRGs; no significant differences in OS and RFS were observed between these two groups $(\mathrm{P}=0.3422$ and $\mathrm{P}=0.6599$; Fig. $2 \mathrm{E}$ and $\mathrm{F}$ ). Among the 75 patients who 
A

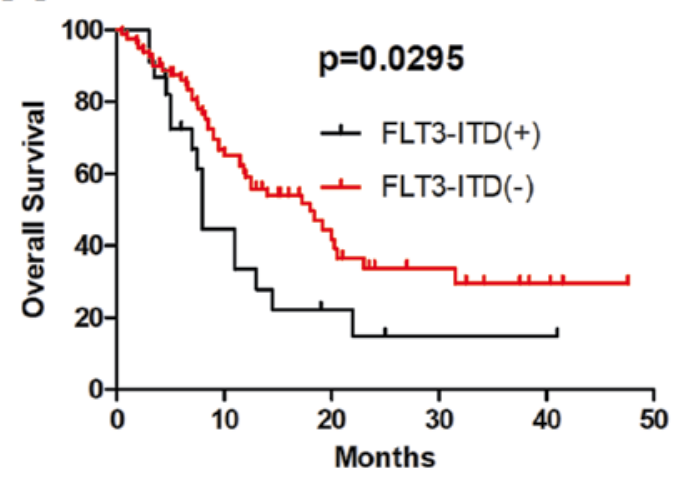

C

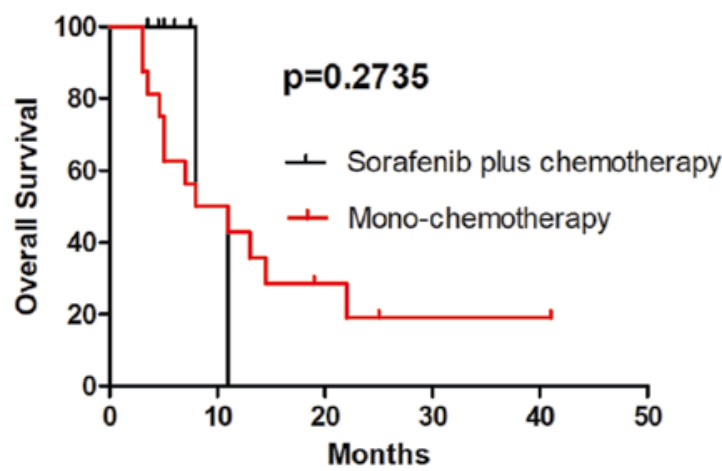

B

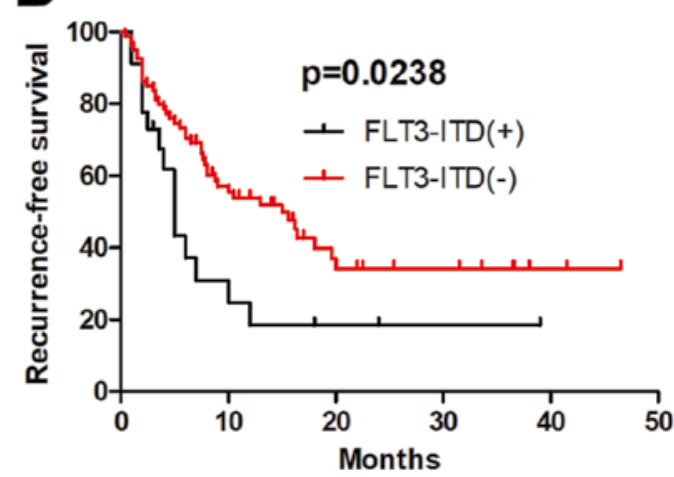

D

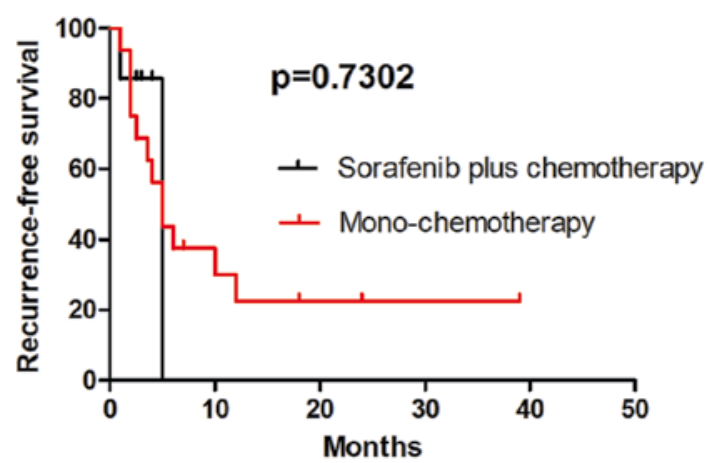

Figure 1. Comparison of OS and RFS in patients with FLT3-ITD-positive and -negative AML. (A) OS and (B) RFS in FLT3-ITD-positive and -negative groups. OS and RFS rates in 23 patients harboring an FLT3-ITD mutation were lower compared with those in FLT3-ITD unmutated patients $(\mathrm{P}=0.0295$ and $\mathrm{P}=0.0238$, respectively). (C) OS and (D) RFS in the mono-chemotherapy and sorafenib plus chemotherapy groups. There was no significant difference in OS and RFS between the mono-chemotherapy and sorafenib plus chemotherapy groups $(\mathrm{P}=0.2735$ and $\mathrm{P}=0.7302$, respectively). OS, overall survival; RFS, recurrence free survival; AML, acute myeloid leukemia; FLT3-ITD, FMS-like tyrosine kinase 3-internal tandem duplication mutation.

received standard treatment, 35 had a single FRG, 7 had a single FLT3-ITD mutation, 11 had an FLT3-ITD mutation and FRGs, 17 had a single DRG, and 5 had a combination of FLT3-ITD mutation and DRGs (Table IV). Compared with patients with a single FLT3-ITD mutation, the OS and RFS were significantly longer in patients with an FLT3-ITD mutation together with FRGs $(\mathrm{P}=0.0361$ and $\mathrm{P}=0.0426$, respectively; Fig. 2A and B). No significant differences in OS and RFS were observed between patients with a single FLT3-ITD mutation and those with DRGs $(\mathrm{P}=0.8549$ and $\mathrm{P}=0.7879$, respectively; Fig. 2C and D).

FLT3-ITD mutations in normal karyotype (NK) and abnormal karyotype (AK). The FLT3-ITD mutation was common in patients with NK. A number of patients with AK also had FLT3-ITD mutations. Out of the 23 patients with an FLT3-ITD mutation were analyzed, 6 were AK and $17 \mathrm{NK}$; no significant differences in OS and RFS were observed between these two groups (Fig. 3A and B). Further analysis revealed that the 3-year OS and RFS were longer in patients with FLT3-ITD combined with NPM1 mutations compared with in patients with a single FLT3-ITD mutation, but there were no statistically significant differences (Fig. 3C and D). This result may be associated with the relatively small number of cases; further large sample studies are therefore required to confirm it.

\section{Discussion}

AML is a heterogeneous disorder of hematopoietic progenitor cells, characterized by blocked differentiation and uncontrolled proliferation (15). Sequencing of AML genomes has revealed that AML is genetically diverse and clonally heterogeneous with multiple mutations, with a majority of patients having $>2$ gene mutations and being clonally represented $(16,17)$. The most frequently mutated genes in AML are NPM1 (25-35\%), CEBPA (6-10\%), RUNX1 (5-15\%), FLT3-ITD ( 20\%), DNMT3A (18-22\%), ASXL1 (5-17\%), KIT (<5\%) and TET2 (7-25\%) (1). AML1-ETO, mutated NPM1 without FLT3-ITD, biallelic mutated $C E B P A$ and $C B F B-M Y H 11$ were associated with a favorable prognosis (1), and $E V I 1, M L L-A F 6, D E K-C A N$ and FLT3-ITD with adverse prognosis. FLT3-ITD is a distinct clinical entity associated with a poor prognosis $(11,18,19)$. In accordance with the results of the present study, AML with FLT3-ITD tends to affect older patients with higher peripheral white blood cell counts, NK (20), shorter remission duration and higher relapse rates compared with patients with FLT3-unmutated AML.

The FLT3-ITD mutation has been revealed to usually coexist with other molecular genetics and cytogenetic alterations; however, the prognostic function of FLT3-ITD combined with other gene mutations or fusion genes is not clear. In the present retrospective study, 103 patients with 
A

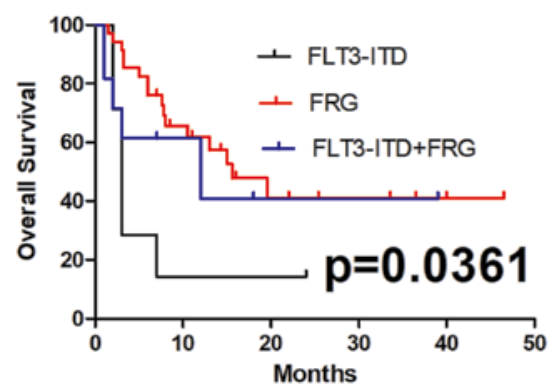

C

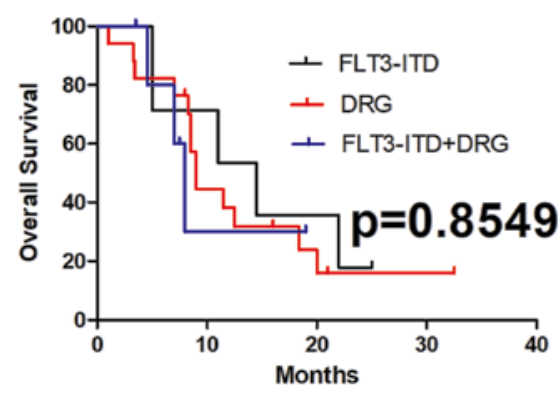

E

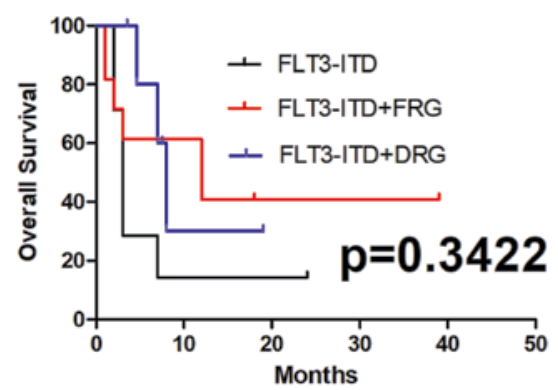

B
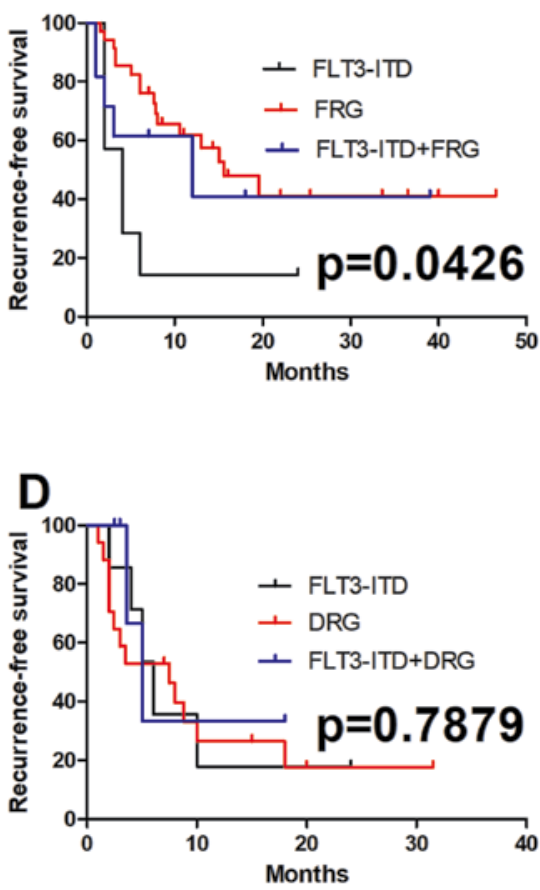

F

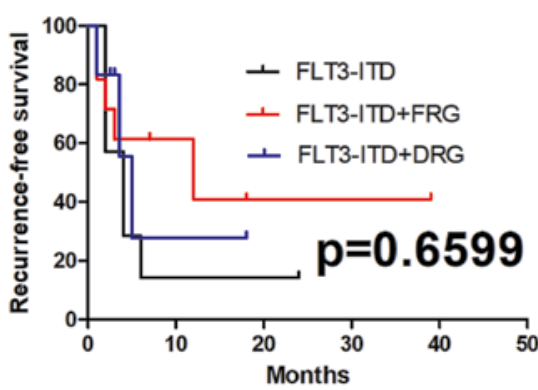

Figure 2. Comparison of OS and RFS between FRGs and DRGs in patients with FLT3-ITD-mutated AML. OS in (A) FRGs alone, (C) DRGs alone or (E) compared with each other, with an FLT3-ITD mutation. OS was significantly longer in patients with an FLT3-ITD mutation combined with FRGs $(\mathrm{P}=0.0361)$. There were no significant differences in OS between the patients with a single FLT3-ITD mutation and the patients with $\mathrm{DRG}$ ( $\mathrm{P}=0.8549)$. RFS in (B) FRGs or (D) DRGs combined with an FLT3-ITD mutation, or (F) the two compared with each other. RFS were significantly longer in patients with an FLT3-ITD mutation combined with FRGs ( $\mathrm{P}=0.0426)$, but no significant differences in RFS were observed between the patients with a single FLT3-ITD mutation and those with DRGs $(\mathrm{P}=0.7879)$. OS, overall survival; RFS, recurrence free survival; FRGs, favorable-risk genes; DRGs, adverse-risk genes; AML, acute myeloid leukemia; FLT3-ITD, FMS-like tyrosine kinase 3-internal tandem duplication mutation.

AML were analyzed, and it was revealed that the OS and RFS rates in 23 patients harboring FLT3-ITD mutations were lower compared with those in patients without FLT3-ITD mutations, but no significant differences in the $\mathrm{CR}$ and $\mathrm{OR}$ rates were observed between these two groups. NPM1 and FLT3-ITD are two of the most common genetic abnormalities in AML. Patients with NPM1 mutations have a favorable prognosis, older patients with NPMI-positive and FLT3-ITD-negative genotype have a relatively favorable prognosis, and patients with an FLT3-ITD mutation have a poor prognosis (21). The prognostic effect of the NPM1 mutation in de novo AML may also be influenced by the relative abundance of the mutated allele (22). FLT3-ITD mutation rates were twice as frequent in NPM1 mutant AML, when compared with AML with wild-type NPMI (22). The FLT3-ITD co-mutation has been revealed to diminish the favorable effect of the NPMI mutation (23). Consistent with previous studies, the prognosis of patients with the FLT3-ITD and NPM1 co-mutation in the present study was improved following high intensity chemotherapy, when compared with the patients with a single FLT3-ITD mutation. However, no statistically significant differences were observed between these two groups, which may be due to the small patient number. FLT3-ITD also co-existed with other genetic abnormalities, including FRGs including CEBPA and AML1-ETO, and DRGs including $N-R A S, N M T 3 A, E V I 1$, DupMLL and HOXI1 (24,25). In the present study, 76 patients with AML with genetic abnormalities were analyzed and the survival of patients with an FLT3-ITD mutation combined with good prognosis genes were compared with those with an FLT3-ITD mutation combined with poor prognosis genes. The results revealed no significant differences in OS and RFS between these two 
A

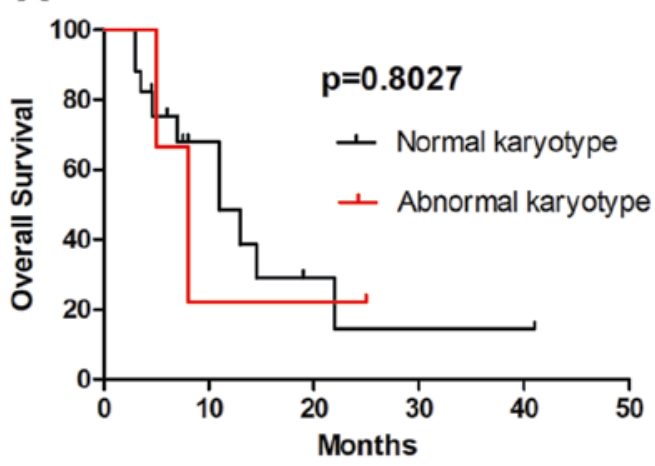

C

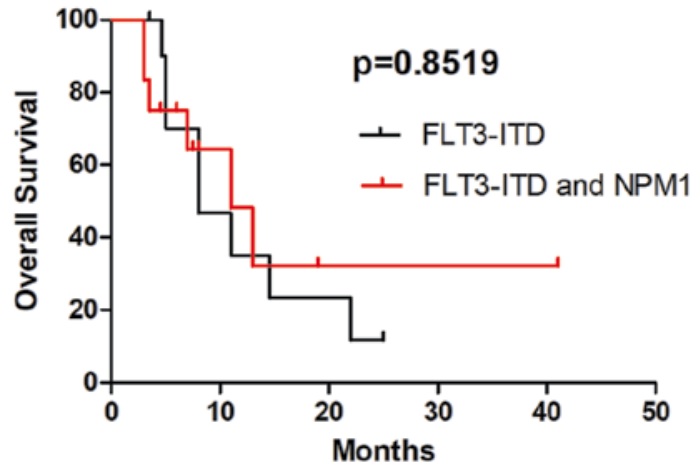

B

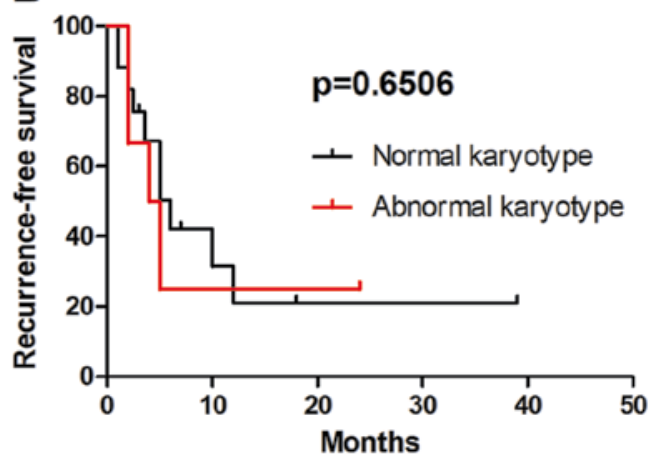

D

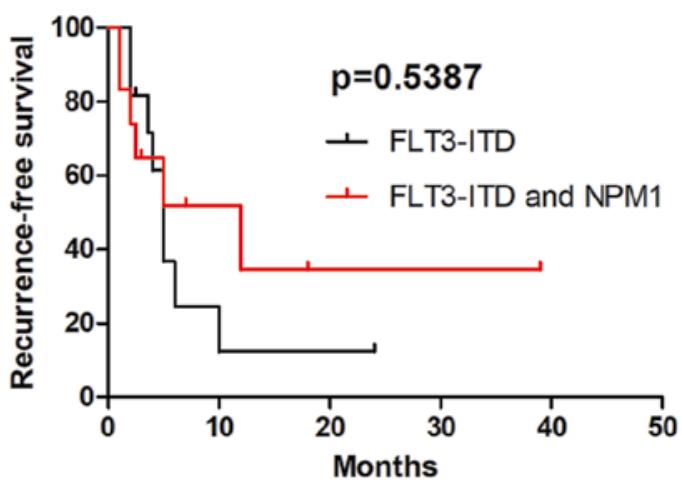

Figure 3. Comparison of OS and RFS in patients with different-karyotype FLT3-ITD-mutated AML. Comparison of (A) OS and (B) RFS between patients with FLT3-ITD-mutated AML with a normal and abnormal karyotype. There were no significant differences in the OS and RFS between these two groups. Comparison of (C) OS and (D) RFS between patients with a FLT3-ITD mono-mutation and FLT3-ITD together with NPM1-mutations. No significant differences in OS and RFS were observed between these two groups. OS, overall survival; RFS, recurrence free survival; AML, acute myeloid leukemia; FLT3-ITD, FMS-like tyrosine kinase 3-internal tandem duplication mutation; NPM1, nucleophosmin 1.

groups, which may be due to the small sample size. Among the 76 patients who received standard treatment, the OS and RFS of patients with an FLT3-ITD mutation combined with FRGs were longer compared with those of patients with a single FLT3-ITD mutation. No significant differences in OS and RFS were observed between the patients with a single FLT3-ITD mutation and those with DRGs.

NK AML accounts for $50 \%$ of all AML and represents the third and largest broad cytogenetic category in AML (26). FLT3-ITD has been recognized as a marker of poor prognosis in patients with NK AML, but FLT3-ITD mutations are also commonly observed in patients with AK AML (27,28), and the prognostic function of the FLT3-ITD mutation in these patients was unclear. A total of 23 FLT3-ITD mutated patients receiving treatment were analyzed; 6 patients were $\mathrm{AK}$ and $17 \mathrm{NK}$, and no significant differences in the OS and RFS were observed between these two groups.

At present, tyrosine kinase inhibitors have been demonstrated to be effective in the treatment of FLT3-mutant AML. The multitargeted kinase inhibitor midostaurin, in combination with chemotherapy, has emerged as a novel standard treatment of patients with FLT3-ITD and TKD-positive AML. It was approved by the FDA for the treatment of AML in $2017(2,29)$. In order to further improve the prognosis of patients with FLT3-mutated AML, selective and potent second-generation FLT3 inhibitors (including gilteritinib, crenolanib, FF-10101 and quizartinib) are being investigated in clinical trials. FF-10101 and quizartinib have each exhibited an excellent efficacy against AML cells and in patients with FLT3 mutations (6,29-32). High-dose daunorubicin or cladribine chemotherapy have also improved clinical outcomes following acquired drug resistance in FLT3-ITD-mutated AML $(3,27)$. However, first-generation FLT3 inhibitors in patients with an FLT3 mutation have resulted in transient reductions in the number of blasts but rarely $\mathrm{CR}$ improvement $(13,14)$. In the present study, 7 patients with an FLT3-ITD mutation received sorafenib plus chemotherapy and exhibited a slightly higher ORR, when compared with patients who were FLT3-ITD-negative; however, no statistically significant difference in OS and RFS was observed between the mono-chemotherapy and sorafenib plus chemotherapy groups. This may be due to the small number of patients that were treated with sorafenib, and future studies should expand the number of cases in order to confirm the conclusions of the present study.

In summary, the FLT3-ITD mutation has been identified as a marker of poor prognosis in AML, and the 
clinical outcomes of patients with FLT3-ITD-mutated AML combined with favorable cytogenetics was improved, when compared with those of patients with FLT3-ITD mono-mutated AML. OS and RFS have been prolonged due to the application of FLT3 inhibitors and adjustments in chemotherapy regimens. However, FLT3 inhibitors may lack a durable and deep response, which is mainly due to acquired drug resistance (3). Allo-HSCT should be performed as early as possible following CR in patients with FLT3-ITD-mutated AML, and maintenance therapy with FLT3 inhibitors may be recommended following allo-HSCT.

In conclusion, the present data demonstrated that favorable-risk cytogenetics may improve the clinical outcomes of patients with FLT3-ITD-mutated AML, but not those of patients with adverse-risk cytogenetic abnormalities. Sorafenib combined with chemotherapy may increase OR rates but could not improve OS and RFS.

\section{Acknowledgements}

Not applicable.

\section{Funding}

The present study was supported by the '333 Projects' Foundation of Jiangsu Province (grant no. BRA2017243), the '533 Projects' Foundation of Huai'an City (grant no. HAA201739), the Science and Technology Fund of Huai'an City (grant no. HAB201810) and the Science and Technology Fund of Jiangsu Commission of Health (grant no. H2018085).

\section{Availability of data and materials}

Not applicable.

\section{Authors' contributions}

ST, CW and LY conceptualized the original idea, designed the experiments and analyzed the data. ST wrote the paper. YC, YD, LS, YS, LL, BD and ZH collected the patient information, treated patients with the regimens and analyzed the data with statistical software. CW and LY revised the manuscript. All authors read and approved the final manuscript.

\section{Ethics approval and consent to participate}

The protocol was reviewed and ethically approved by the Institutional Review Committee of Huai'an No. 1 People's Hospital (Huai'an, China).

\section{Patients consent for publication}

A written informed consent form was obtained from each patient.

\section{Competing interests}

The authors declare that they have no competing interests.

\section{References}

1. Döhner H, Weisdorf DJ and Bloomfield CD: Acute myeloid leukemia. N Engl J Med 373: 1136-1152, 2015.

2. Stone RM, Mandrekar SJ, Sanford BL, Laumann K, Geyer S, Bloomfield CD, Thiede C, Prior TW, Döhner K, Marcucci G, et al: Midostaurin plus chemotherapy for acute myeloid leukemia with a FLT3 mutation. N Engl J Med 377: 454-464, 2017.

3. Choi EJ, Lee JH, Lee JH, Park HS, Ko SH, Hur EH, Moon J, Goo BK, Kim Y, Seol M, et al: Comparison of anthracyclines used for induction chemotherapy in patients with FLT3-ITD-mutated acute myeloid leukemia. Leuk Res 68: 51-56, 2018.

4. Liu Y, He P, Liu F, Shi L, Zhu H, Zhao J, Wang Y, Cheng X and Zhang M: Prognostic significance of NPM1 mutations in acute myeloid leukemia: A meta-analysis. Mol Clin Oncol 2: 275-281, 2014.

5. Boddu P, Kantarjian H, Borthakur G, Kadia T, Daver N, Pierce S, Andreeff M, Ravandi F, Cortes J and Kornblau SM: Co-occurrence of FLT3-TKD and NPM1 mutations defines a highly favorable prognostic AML group. Blood Adv 1: 1546-1550, 2017.

6. Yamaura T, Nakatani T, Uda K, Ogura H, Shin W, Kurokawa N, Saito K, Fujikawa N, Date T, Takasaki M, et al: A novel irreversible FLT3 inhibitor, FF-10101, shows excellent efficacy against AML cells with FLT3 mutations. Blood 131: 426-438, 2018.

7. Vardiman JW, Thiele J, Arber DA, Brunning RD, Borowitz MJ, Porwit A, Harris NL, Le Beau MM, Hellström-Lindberg E, Tefferi A and Bloomfield CD: The 2008 revision of the World Health Organization (WHO) classification of myeloid neoplasms and acute leukemia: Rationale and important changes. Blood 114: 937-951, 2009.

8. Pallisgaard N, Hokland P, Riish øj DC, Pedersen B and Jørgensen P: Multiplex reverse transcription-polymerase chain reaction for simultaneous screening of 29 translocations and chromosomal aberrations in acute leukemia. Blood 92: 574-588, 1998.

9. Yang YL, Lin SR, Chen JS, Hsiao CC, Lin KH, Sheen JM, Cheng CN, Wu KH, Lin SW, Yu SL, et al: Multiplex reverse transcription-polymerase chain reaction as diagnostic molecular screening of 4 common fusion chimeric genes in Taiwanese children with acute lymphoblastic leukemia. J Pediatr Hematol Oncol 32: e323-e330, 2010.

10. Gou H, Zhou J, Ye Y, Hu X, Shang M, Zhang J, Zhao Z, Peng W, Zhou Y, Zhou Y, et al: The prevalence and clinical profiles of FLT3-ITD, FLT3-TKD, NPM1, C-KIT, DNMT3A, and CEBPA mutations in a cohort of patients with de novo acute myeloid leukemia from southwest China. Tumour Biol 37: 7357-7370, 2016.

11. Zhu Y, Zhao H, Zhang X, Wu Y, Xie Y, Li Y, Lian Y, Huang J, Li J, Chen Y and Qian S: Decitabine prior to low-dose cytarabine-based chemotherapy combined with HLA-mismatched stem cell micro-transplantation improved outcomes in elderly patients with newly diagnosed acute myeloid leukaemia. Biol Blood Marrow Transplant 23: 830-835, 2017.

12. Kottaridis PD, Gale RE, Frew ME, Harrison G, Langabeer SE, Belton AA, Walker H, Wheatley K, Bowen DT, Burnett AK, et al: The presence of a FLT3 internal tandem duplication in patients with acute myeloid leukemia (AML) adds important prognostic information to cytogenetic risk group and response to the first cycle of chemotherapy: Analysis of 854 patients from the United Kingdom Medical Research Council AML 10 and 12 trials. Blood 98: 1752-1759, 2001.

13. Stone RM, DeAngelo DJ, Klimek V, Galinsky I, Estey E, Nimer SD, Grandin W, Lebwohl D, Wang Y, Cohen P, et al: Patients with acute myeloid leukemia and an activating mutation in FLT3 respond to a small-molecule FLT3 tyrosine kinase inhibitor, PKC412. Blood 105: 54-60, 2005.

14. Smith BD, Levis M, Beran M, Giles F, Kantarjian H, Berg K, Murphy KM, Dauses T, Allebach J and Small D: Single-agent CEP-701, a novel FLT3 inhibitor, shows biologic and clinical activity in patients with relapsed or refractory acute myeloid leukemia. Blood 103: 3669-3676, 2004.

15. Kindler T, Lipka DB and Fischer T: FLT3 as a therapeutic target in AML: Still challenging after all these years. Blood 116: 5089-5102, 2010.

16. Ding L, Ley TJ, Larson DE, Miller CA, Koboldt DC, Welch JS, Ritchey JK, Young MA, Lamprecht T, McLellan MD, et al: Clonal evolution in relapsed acute myeloid leukaemia revealed by whole-genome sequencing. Nature 481: 506-510, 2012. 
17. Shlush LI, Mitchell A, Heisler L, Abelson S, Ng SWK, TrotmanGrant A, Medeiros JJF, Rao-Bhatia A, Jaciw-Zurakowsky I, Marke R, et al: Tracing the origins of relapse in acute myeloid leukaemia to stem cells. Nature 547: 104-108, 2017.

18. Fröhling S, Schlenk RF, Breitruck J, Benner A, Kreitmeier S, Tobis K, Döhner H, Döhner K and AML Study Group Ulm: Acute myeloid leukemia: Prognostic significance of activating FLT3 mutations in younger adults (16 to 60 years) with acute myeloid leukemia and normal cytogenetics: A study of the AML Study Group Ulm. Blood 100: 4372-4380, 2002.

19. Thiede C, Steudel C, Mohr B, Schaich M, Schäkel U, Platzbecker U, Wermke M, Bornhäuser M, Ritter M, Neubauer A, et al: Analysis of FLT3-activating mutations in 979 patients with acute myelogenous leukemia: Association with FAB subtypes and identification of subgroups with poor prognosis. Blood 99: 4326-4335, 2002.

20. Leick MB and Levis MJ: The future of targeting FLT3 activation in AML. Curr Hematol Malig Rep 12: 153-167, 2017.

21. Ostronoff F, Othus M, Lazenby M, Estey E, Appelbaum FR, Evans A, Godwin J, Gilkes A, Kopecky KJ, Burnett A, et al: Prognostic significance of NPM1 mutations in the absence of FLT3-internal tandem duplication in older patients with acute myeloid leukemia: A SWOG and UK national cancer research institute/medical research council report. J Clin Oncol 33: 1157-1164, 2015.

22. Patel SS, Kuo FC, Gibson CJ, Steensma DP, Soiffer RJ, Alyea EP 3rd, Chen YA, Fathi AT, Graubert TA, Brunner AM, et al: High NPM1-mutant allele burden at diagnosis predicts unfavorable outcomes in de novo AML. Blood 131: 2816-2825, 2018.

23. Döhner H, Estey E, Grimwade D, Amadori S, Appelbaum FR, Büchner T, Dombret H, Ebert BL, Fenaux P, Larson RA, et al: Diagnosis and management of AML in adults: 2017 ELN recommendations from an international expert panel. Blood 129: 424-447, 2017.

24. Loghavi S, Zuo Z, Ravandi F, Kantarjian HM, Bueso-Ramos C, Zhang L, Singh RR, Patel KP, Medeiros LJ, Stingo F, et al: Clinical features of de novo acute myeloid leukemia with concurrent DNMT3A, FLT3 and NPM1 mutations. J Hematol Oncol 7: $74,2014$.
25. Bur illo-Sanz S, Morales-Camacho RM, Caballero-Velázquez T, Vargas MT, García-Lozano JR, Falantes JF, Prats-Martín C, Bernal R, and Pérez-Simón JA: NUP98-HOXA9 bearing therapy-related myeloid neoplasm involves myeloid-committed cell and induces HOXA5, EVI1, FLT3, and MEIS1 expression. Int J Lab Hematol 38: 64-71, 2016.

26. Cagnetta A, Adamia S, Acharya C, Patrone F, Miglino M, Nencioni A, Gobbi $\mathbf{M}$ and Cea M: Role of genotype-based approach in the clinical management of adult acute myeloid leukemia with normal cytogenetics. Leuk Res 38: 649-659, 2014.

27. Mori $M$ and Spraque J: The successful remission induction by sorafenib and long-term complete remission in a FLT3-ITD-positive patient with a refractory acute erythroid leukemia and abnormal cytogenetics. Leuk Res 36: e1-e3, 2012.

28. Libura M, Giebel S, Piatkowska-Jakubas B, Pawelczyk M, Florek I, Matiakowska K, Jazwiec B, Borg K, Solarska I, Zawada M, et al: Cladribine added to daunorubicin-cytarabine induction prolongs survival of FLT3-ITD ${ }^{+}$normal karyotype AML patients. Blood 127: 360-262, 2016

29. Stein EM: FLT3 inhibitors for relapsed or refractory acute myeloid leukaemia. Lancet Oncol 19: 845-850, 2018.

30. Cortes JE, Tallman MS, Schiller GJ, Trone D, Gammon G, Goldberg SL, Perl AE, Marie JP, Martinelli G, Kantarjian HM and Levis MJ: Phase 2b study of two dosing regimens of quizartinib monotherapy in FLT3-ITD mutated, relapsed or refractory AML. Blood 132: 598-607, 2018.

31. Quizartinib bests chemo for FLT3-mutant AML. Cancer Discov 8: OF2, 2018.

32. Cortes J, Perl AE, Döhner H, Kantarjian H, Martinelli G, Kovacsovics T, Rousselot P, Steffen B, Dombret H, Estey E, et al: Quizartinib, an FLT3 inhibitor, as monotherapy in patients with relapsed or refractory acute myeloid leukaemia: an open-label, multicentre, single-arm, phase 2 trial. Lancet Oncol 19: 889-903, 2018.

This work is licensed under a Creative Commons Attribution-NonCommercial-NoDerivatives 4.0 International (CC BY-NC-ND 4.0) License. 